\title{
Multiple sparse volumetric priors for distributed EEG source reconstruction
}

\author{
Gregor Strobbe ${ }^{\mathrm{a}, \mathrm{f}, *}$, Pieter van Mierlo ${ }^{\mathrm{a}, \mathrm{f}}$, Maarten De Vos ${ }^{\mathrm{b}, \mathrm{c}, \mathrm{d}}$, Bogdan \\ Mijoviće,f $^{\text {,f }}$ Hans Hallez ${ }^{\mathrm{g}}$, Sabine Van Huffel ${ }^{\mathrm{e}, \mathrm{f}}$, José David López ${ }^{\mathrm{h}}$, Stefaan \\ Vandenberghe $^{\mathrm{a}}$ \\ ${ }^{a}$ Ghent University - iMinds, Department of Electronics and Information Systems, \\ MEDISIP, De Pintelaan 185, Building BB floor 5, 9000, Ghent, Belgium \\ ${ }^{b}$ University of Oldenburg, Methods in Neurocognitive Psychology, Department of \\ Psychology, 26111 Oldenburg, Germany \\ ${ }^{c}$ University of Oldenburg, Research Center Neurosensory Science, 26111 Oldenburg, \\ Germany \\ ${ }^{d}$ University of Oldenburg, Cluster of Excellence Hearing4all, 26111 Oldenburg, Germany \\ ${ }^{e} K U$ Leuven, Department of Electrical Engineering-ESAT, STADIUS Centre for \\ Dynamical Systems, Signal Processing and Data Analytics, Kasteelpark Arenberg 10, \\ 3001 Leuven, Belgium \\ ${ }_{i \text { Minds Medical IT department, Belgium }}$ \\ ${ }^{g}$ Catholic University College of Bruges-Ostend, Faculty of Engineering Technology, \\ Electronics/ICT, Zeedijk 101, 8400, Ostend, Belgium \\ ${ }^{h}$ SISTEMIC, Department of Electronic Engineering, Universidad de Antioquia UDEA. \\ Calle 70 No. 52-21,Medellín, Colombia
}

\section{Abstract}

We revisit the multiple sparse priors (MSP) algorithm implemented in the statistical parametric mapping software (SPM) for distributed EEG source reconstruction (Friston et al., 2008). In the present implementation, multiple cortical patches are introduced as source priors based on a dipole source

\footnotetext{
*Corresponding author - phone number: +32484777651

Email addresses: gregor.strobbe@ugent.be (Gregor Strobbe), pieter.vanmierlo@ugent.be (Pieter van Mierlo), maarten.de.vos@uni-oldenburg.de (Maarten De Vos), bogdan.mijovic@esat.kuleuven.be (Bogdan Mijović), hans.hallez@kuleuven.be (Hans Hallez), sabine.vanhuffel@esat.kuleuven.be (Sabine Van Huffel), josedavid@udea.edu.co (José David López), stefaan.vandenberghe@ugent.be (Stefaan Vandenberghe)
} 
space restricted to a cortical surface mesh. In this note, we present a technique to construct volumetric cortical regions to introduce as source priors by restricting the dipole source space to a segmented gray matter layer and using a region growing approach. This extension allows to reconstruct brain structures besides the cortical surface and facilitates the use of more realistic volumetric head models including more layers, such as cerebrospinal fluid (CSF), compared to the standard 3-layered scalp-skull-brain head models. We illustrated the technique with ERP data and anatomical MR images in 12 subjects. Based on the segmented gray matter for each of the subjects, cortical regions were created and introduced as source priors for MSP-inversion assuming two types of head models. The standard 3-layered scalp-skullbrain head models and extended 4-layered head models including CSF. We compared with the current implementation by assessing the free energy corresponding with each of the reconstructions using Bayesian model selection for group studies. Strong evidence was found in favor of the volumetric MSP approach compared to the MSP approach based on cortical patches for both types of head models. Overall, the strongest evidence was found in favor of the volumetric MSP reconstructions based on the extended head models including CSF. These results were verified by comparing the reconstructed activity. The use of volumetric cortical regions as source priors is a useful complement to the present implementation as it allows to introduce more complex head models and volumetric source priors in future studies.

Keywords: electroencephalography, multiple sparse priors, volumetric sparse priors, Bayesian model comparison, statistical Parametric Mapping 


\section{Introduction}

In this note we present a new application of hierarchical or empirical Bayes for distributed EEG source reconstruction. We depart from the parametric empirical Bayesian (PEB) framework used in the Statistical Parametric Mapping software (SPM) package (Wellcome Trust Centre for Neuroimaging, London, UK). Within the framework, the multiple sparse priors (MSP) algorithm is the state-of-the-art inverse technique. Depending on the EEG data, the algorithm allows the automatic selection of multiple cortical sources with compact spatial support that are specified in terms of empirical priors (Friston et al., 2008).

In the present implementation of the MSP algorithm, multiple cortical patches of sources are constructed based on a source space of dipoles constrained to a cortical surface mesh (Mattout et al., 2007) and the field propagation of the surface patches is calculated based on a 3-layered scalp-skullbrain head model (Henson et al., 2009). Constraining the dipolar sources to a cortical mesh does not allow the reconstruction of brain activity besides the cortical surface. Moreover, it is not straightforward to use more complex head models that extend the 3-layered model with extra layers such as cerebrospinal fluid (CSF). Because the dipoles are located on the boundary between the CSF and the brain, they will either be located inside the CSF or brain compartment which does not satisfy the restrictions to the source space of commonly used numerical methods, such as the boundary element method (Mosher et al., 1999), finite difference method (Vanrumste et al., 2001; Hallez et al., 2005) or finite element method (Wolters et al., 2002), to properly calculate the dipole field propagation (Stenroos \& Nenonen, 2012; 
Strobbe et al., 2014).

In this work, we propose a technique to construct volumetric regions based on a dipole source space restricted to gray matter, segmented from an anatomical MR image, and using a region growing technique. This approach allows the inclusion of more prior information about the anatomy and shape of the sources and does not require the extraction of the cortical surface. It opens up the possibility to use the MSP algorithm to reconstruct brain structures besides the cortical surface and facilitates the use of more realistic volumetric head models including cerebrospinal fluid (CSF) compared to the currently used 3-layered scalp-skull-brain head models.

To illustrate the volumetric MSP approach, we used realistic ERP datasets and anatomical MR images in 12 subjects. Based on the segmented gray matter for each of the subjects, cortical regions were created and introduced as source priors for MSP-inversion assuming two types of head models. For every subject, a 3-layered volumetric subject specific head model was constructed. Also extended 4-layered head models including CSF were built to investigate the influence of increasing the head model complexity. We compared with the present implementation by assessing the free energy corresponding with the reconstructions using Bayesian model selection for group studies (Stephan et al., 2009; Rigoux et al., 2013). The reconstructed activity was also compared with the results of previous studies using similar ERP datasets (Mijović et al., 2012).

In the first section of this paper, we will briefly present the PEB framework and the MSP algorithm. We will explain how we extended the currently used approach based on cortical patches to volumetric regions and 
subsequently describe how the different head models used in this study were constructed. Next, we explain how we compared the models using Bayesian model selection and verified the reconstructed activity. We conclude with a discussion of the benefits and potential of using volumetric source priors.

\section{Methods}

\subsection{Distributed EEG source reconstruction}

Assume we represent the EEG measurements as a multivariate linear model involving a distributed source model with fixed positions and orientations (Dale \& Sereno, 1992):

$$
V=L J+\epsilon
$$

where $V \in \mathbb{R}^{N_{c} \times N_{t}}$ is the EEG dataset of $N_{c}$ channels and $N_{t}$ time samples, $J \in \mathbb{R}^{N_{d} \times N_{t}}$ the amplitude of $N_{d}$ current dipoles with fixed orientations,

$\epsilon \in \mathbb{R}^{N_{c} \times N_{t}}$ is zero mean Gaussian noise and $L \in \mathbb{R}^{N_{c} \times N_{d}}$ is the lead field matrix linking the source amplitudes in $J$ to the electrical scalp potentials in $V$. The lead field matrix represents the forward model and embodies assumptions about the head model and the forward modeling technique that is used.

Because of the ill-posed nature of the EEG source reconstruction problem (Baillet \& Garnero, 1997), we need to add prior information to find a unique solution. There are different techniques that allow this, such as the weighted minimum norm (WMN) solution (Hämäläinen \& Ilmoniemi, 1994): 


$$
\widehat{J}=\min _{J}\left(\left\|C_{\epsilon}^{-1 / 2}(L J-V)\right\|^{2}+\lambda\|W J\|^{2}\right)
$$

This approach implicates minimizing an energy function, with $C_{\epsilon}$ the prior covariance of the sensor noise, $W$ a weighting matrix including prior information of the source activity and with $\lambda$ a hyperparameter that tunes the relative importance of the accuracy of the model $\left\|C_{\epsilon}^{-1 / 2}(L J-V)\right\|^{2}$, and the regularisation term $\|W J\|^{2}$. Given that $\left(\lambda W^{T} W\right)^{-1}=C_{J}$, with $C_{J}$ the prior covariance of the sources (i.e., it embodies our assumptions about the interaction among the sources), the solution of this equation becomes (Phillips et al., 2005; Grech et al., 2008):

$$
\widehat{J}=\left(C_{J}\right) L^{T}\left[L\left(C_{J}\right)^{-1} L^{T}+C_{\epsilon}\right]^{-1} V
$$

It follows that the solution of Eq.(3) directly depends on $C_{\epsilon}$ and $C_{J}$.

\subsection{Parametric empirical Bayes: multiple priors}

Eq.(1) can also be expressed in the context of a two-level hierarchical parametric empirical Bayesian (PEB) model:

$$
\begin{array}{r}
V=L J+\epsilon_{1} \\
J=\epsilon_{2}
\end{array}
$$

with $\epsilon_{1}$ and $\epsilon_{2}$ assumed to follow a Gaussian distribution with zero mean: $\epsilon_{1} \sim N\left(0, C_{\epsilon}\right)$ and $\epsilon_{2} \sim N\left(0, C_{J}\right)$. The covariance matrices $C_{e}$ and $C_{J}$ can be modeled as a linear combination of covariance components (Phillips et al., 2007): 


$$
\begin{aligned}
& C_{\epsilon}=\lambda_{1}^{(1)} Q_{1}^{(1)}+\lambda_{2}^{(1)} Q_{2}^{(1)}+\ldots \\
& C_{J}=\lambda_{1}^{(2)} Q_{1}^{(2)}+\lambda_{2}^{(2)} Q_{2}^{(2)}+\ldots
\end{aligned}
$$

With $\lambda_{1}^{(1)}, \lambda_{2}^{(1)}, \ldots$ and $\lambda_{1}^{(2)}, \lambda_{2}^{(2)}, \ldots$, the hyperparameters that balance the various covariance components either at the first (sensor) or second (source) level (Phillips et al., 2005).

In the SPM - PEB framework the hyperparameters are estimated using a variational Bayesian estimation scheme by optimizing the free energy (Friston et al., 2007) given the covariance components. As such, $C_{\epsilon}\left(\mu_{1}\right)$ and $C_{J}\left(\mu_{2}\right)$, with $\mu_{1}=\left\{\lambda_{i}^{(1)}\right\}$ with $i=1,2, \ldots$ and $\mu_{2}=\left\{\lambda_{i}^{(2)}\right\}$ with $i=1,2, \ldots$, can be calculated. It follows that the expectation of the source intensities $J$ given $V$ is equal to:

$$
E[J]=C_{J}\left(\mu_{2}\right) L^{T}\left[L C_{J}\left(\mu_{2}\right) L^{T}+C_{\epsilon}\left(\mu_{1}\right)\right]^{-1} V
$$

With $E[J]$ the expected value of $J$. Note that we obtain the same solution as in Eq. (3), with the difference that we can introduce multiple constraints or priors in the form of covariance components.

\subsection{Multiple sparse priors algorithm}

In absence of prior information, we assume the same amount of prior variance on all sensors: $C_{\epsilon}=\lambda_{1}^{(1)} I_{N_{c}}$, where $I_{N_{c}} \in \mathbb{R}^{N_{c} \times N_{c}}$ is an identity matrix, and $\lambda_{1}^{(1)}$ is the sensor noise variance.

In the multiple sparse priors (MSP) algorithm (Friston et al., 2008), a weighted sum of $N_{p}$ predefined source covariance candidate matrices is used, where each covariance matrix represents a potential activated area of cortex: 


$$
C_{J}=\sum_{i=1}^{N_{p}}\left(\lambda_{i}^{(2)}\right) Q_{i}^{(2)}
$$

The hyperparameters $\left\{\lambda_{1}^{(2)}, \ldots, \lambda_{N_{p}}^{(2)}\right\}$ weight these covariance components and control the power allocated to each of them. Note that these components may embody different types of informative priors, e.g., different smoothing functions, medical knowledge, fMRI priors (Henson et al., 2011).

\subsubsection{Multiple sparse priors (MSP) based on cortical surface mesh}

In the currently used MSP approach implemented in the SPM software package, the dipole source space is constrained to the nodes of a cortical surface mesh. Based on this surface mesh, covariance components $\left\{Q_{1}^{(2)}, Q_{2}^{(2)} \ldots, C_{N_{p}}^{(2)}\right\}$ are constructed that are each corresponding to a different locally smooth focal patch of cortex. The covariance components are constructed using the columns of a Green's function, $Q_{G} \in \mathbb{R}^{N_{d} \times N_{d}}$ defined as:

$$
Q_{G}=\exp \left(\sigma G_{L}\right)
$$

and calculated based on a graph Laplacian $G_{L} \in \mathbb{R}^{N_{d} \times N_{d}}$, with inter-dipole connectivity information containing the neighboring dipoles, and $\sigma$, a positive constant value that determines the smoothness of the current distribution or spatial extent of the activated regions (Friston et al., 2008). The graph Laplacian $G_{L}$ is calculated using an adjacency matrix corresponding with the vertices and faces provided by the cortical surface mesh.

As such, each column of $Q_{G}$ corresponds with a cortical patch and has a bell shape, with a full width half maximum depending on the neighboring dipoles and the smoothing factor $\sigma$ which is equal to 0.6 in the SPM software. 
The centers of these patches correspond to the original set of dipoles used to form the lead field matrix.

\subsubsection{Extension to multiple sparse volumetric priors (MSVP)}

In the construction process of the surface patches, a cortical mesh is used to calculate the adjacency matrix using the faces and vertices of the cortical mesh. Therefore the connections between the sulci of gray matter are automatically taken into account. In order to incorporate the shape of the cortical layer based on the segmented gray matter, we propose a technique to construct volumetric sparse regions using a region growing approach.

Assume a source space of distributed dipoles located inside the segmented gray matter. For each dipole in the source space, a volumetric region is determined based on region growing within the segmented gray matter. The region growing approach starts from a dipole and is characterized by a certain maximum distance to the dipole. As such, the neighboring dipoles for each dipole of the source space can be determined as the dipoles located within the corresponding region of each dipole. An example of the approach in 2D is given in Fig. 1. In this example, we assume that we have a slice of a cubic $3 \mathrm{D}$ volumetric head model $(1 \times 1 \times 1 \mathrm{~mm}$ resolution $)$ with dipoles in the segmented gray matter equidistant to each other, with a $3 \mathrm{~mm}$ spacing.

With the information of the neighboring dipoles within the region of each dipole, the adjacency matrix, graph laplacian and Green's function can be calculated. Each column of the Green's function $Q_{G}$ represents a volumetric region and has a bell shape, with a full width half maximum depending on the maximum distance to the original dipole used in the region growing approach and the smoothing factor $\sigma$. 

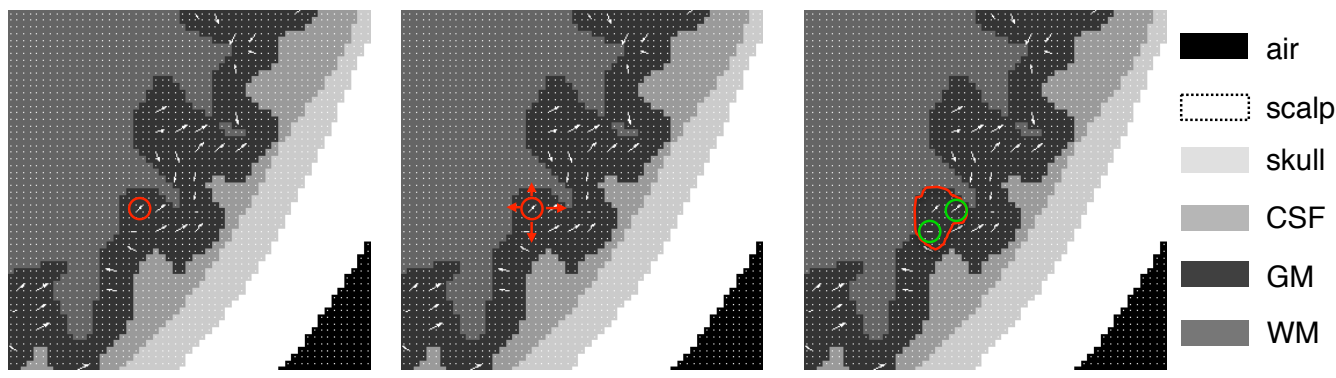

Figure 1: An example of the used region growing approach in 2D. The original dipole from which the region growing starts is depicted by the red circle on the left. The maximum distance to the original dipole is $5 \mathrm{~mm}$ (i.e. 5 voxels). Based on this restriction, the original dipole has 2 neighboring dipoles depicted by the green circles on the right. CSF $=$ cerebrospinal fluid, $\mathrm{GM}=$ gray matter, $\mathrm{WM}=$ white matter

\subsection{Illustration on realistic data}

To illustrate the proposed approach, we used ERP data and anatomical MR images in 12 subjects to which checkerboard stimuli were presented. The whole data-acquisition process and preprocessing of the data is described in detail in Mijović et al. (2012). In brief, twelve healthy individuals performed 80 trials of circular black-and-white checkerboard stimuli presented to one of the four quadrants of the visual field. The EEG data were collected from 62 electrodes using the BrainAmp MR+ system (BrainProducts, Gliching, Germany). To extract task-related ERPs, data were then segmented from -100 ms before until $500 \mathrm{~ms}$ after stimulus onset, and baseline corrected. Finally, the data were average referenced resulting in $12 \times 4$ different grand averaged ERP datasets corresponding with each condition: down left, down right, upper left or upper right.

In addition, full brain anatomical images were obtained with the mag- 
netization prepared rapid gradient echo (MPRAGE) imaging sequence (230 coronal slices, time to echo $[\mathrm{TE}]=4.6 \mathrm{~ms}, \mathrm{TR}=9.7 \mathrm{~s}$ ) which we used to construct the subject specific head models (see Section 2.4.1).

\subsubsection{Construction of head models}

Because we used a volumetric finite difference method based on reciprocity (FDRM) (Hallez et al., 2005; Strobbe et al., 2014) to calculate the dipole field propagation, each of the considered head models was volumetric with a $3 \mathrm{D}, 1 \times 1 \times 1 \mathrm{~mm}$ voxel resolution.

\section{3-layered models}

We followed the default approach implemented in the SPM software to construct subject specific 3-layered models based on the anatomical MR images of the subjects. Meshes representing the scalp, outer skull and inner skull were extracted from the subjects' MR images (see Fig. 2A). These meshes were subsequently converted to filled volumes in order to construct volumetric 3-layered head models. Isotropic conductivities of the brain, scalp layer and skull layer were set to $0.33 \mathrm{~S} / \mathrm{m}, 0.33 \mathrm{~S} / \mathrm{m}$ and $0.022 \mathrm{~S} / \mathrm{m}$ (Oostendorp et al., 2000) respectively. In Fig. 2B an example is given of a 3-layered volumetric model, denoted as 3lay. We coregistered the 62 electrode positions, corresponding with our realistic EEG data, to the scalp surface and embedded them inside the boundary of the scalp.

\section{4-layered models}

Using the anatomical MR images of the subjects, we segmented gray matter, white matter and CSF using SPM8 segmentation techniques (Ashburner 
\& Friston, 2003). Based on these segmentations and the 3-layered models, 4-layered head models were constructed including a brain and CSF compartment. The conductivity of the CSF was set to $1.79 \mathrm{~S} / \mathrm{m}$ (Baumann et al., 1997 ) and $0.33 \mathrm{~S} / \mathrm{m}$ for the brain layer. In Fig. 2C an example is given of a 4layered volumetric model, denoted as 4lay. We coregistered the 62 electrode positions, corresponding with our realistic EEG data, to the scalp surface and embedded them inside the boundary of the scalp.

\subsubsection{Construction of dipole source spaces}

\section{Cortical surface mesh}

For each subject, the source space was constructed using a canonical cortical mesh, defined in a standard stereotactic space. This mesh was warped, in a nonlinear fashion, to match the subjects' anatomy (Mattout et al., 2007). This resulted in 8196 dipoles distributed on the nodes of the warped cortical surface mesh, with fixed orientations orthogonal to the mesh.

\section{Volumetric}

For each of the models the source space was constructed based on the segmented gray matter. Dipoles were assumed inside gray matter on a cubic grid equidistant to each other with a spacing of $3 \mathrm{~mm}$. Because the dipole model in the FDRM method extends over 3 nodes of the voxel elements in the $\mathrm{x}, \mathrm{y}$ and $\mathrm{z}$ direction (Hallez et al., 2005), we ensured that at least 2 voxels of gray matter were between the central node of the dipole model and the boundaries with other tissues in the $\mathrm{x}, \mathrm{y}$ and $\mathrm{z}$ direction. This resulted in approximately 10.000 dipoles for each of the models. The orientations of the dipoles were determined based on a method described in (Phillips et al., 

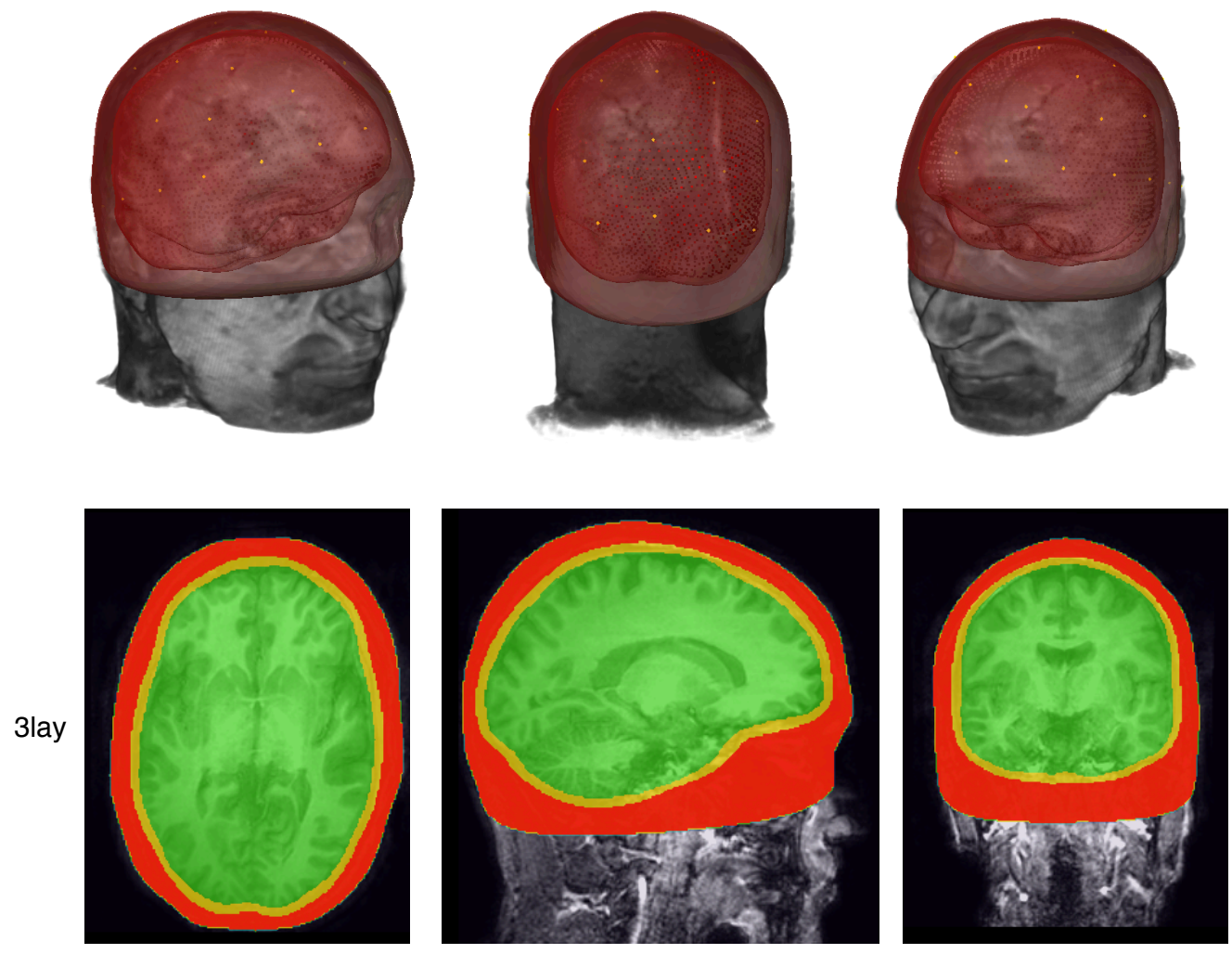

scalp

skull

brain

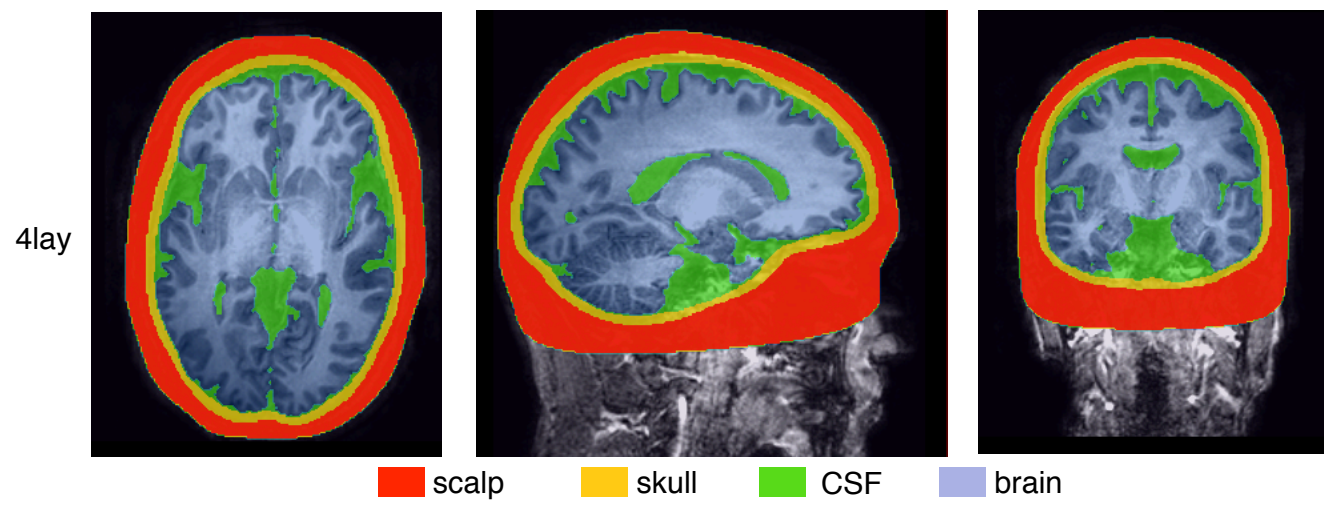

Figure 2: Example of the subject specific head models constructed based on an anatomical MR image. The subject specific anatomical MR image is depicted in gray. The electrodes are depicted in yellow. In the first row, the surface meshes used to construct the 3layered models in SPM are shown in red. The inner mesh corresponding with the red dots corresponds with the inner skull surface. The second row depicts 3 orthogonal slices of the 3-layered volumetric model denoted as 3lay. The third row depicts 3 orthogonal slices of the 4-layered volumetric model including CSF denoted as 4lay. 
2002). This method starts from the segmented white matter to calculate the orientations of the grid points in gray matter after smoothing and taking the gradient of the volume. An example is given in Fig. 3.
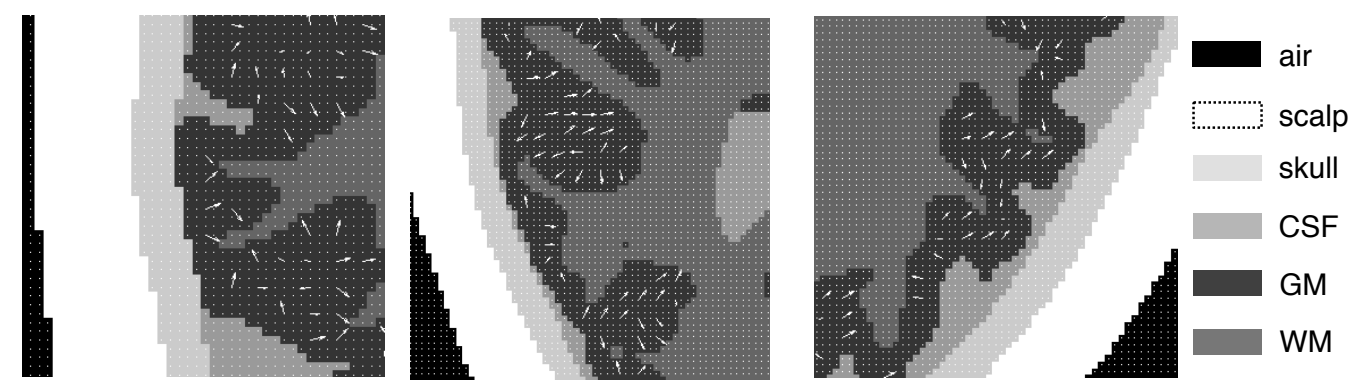

Figure 3: Example of the dipole orientations depicted by the white arrows shown in a detailed view corresponding with 3 orthogonal slices. The different colors in the slices correspond with different tissue types shown in the legend on the right. CSF $=$ cerebrospinal fluid, GM = gray matter, $\mathrm{WM}=$ white matter.

\subsubsection{Construction of the source priors}

\section{Cortical surface}

We considered a subset of $N_{p}=256$ source priors, sampled from the total set of priors (or columns of $Q_{G}$ ), covering the entire cortical surface mesh. The size of each patch was approximately $1 \mathrm{~cm}^{2}$ (dependent on the distance to the nearest dipoles). This type of source priors is denoted as Surf.

\section{Volumetric}

Again a set of $N_{p}=256$ volumetric regions was considered covering the entire cortical layer. The maximum distance to the original dipole and the smoothing factor $\sigma$ were set to $5 \mathrm{~mm}$ and 0.6 respectively. The size of the 
regions was therefore approximately $1 \mathrm{~cm}^{3}$. In order to compare with the default approach, we chose the centers of the 256 volumetric regions as close as possible to the centers of the 256 surface patches. All distances between the centers were below $9 \mathrm{~mm}$. This type of source priors is denoted as Vol.

\subsubsection{Bayesian model comparison based on free energy}

In order to compare the different models we applied Bayesian model selection based on free energy for group studies (Stephan et al., 2009; Rigoux et al., 2013). For all the comparisons reported in this study, we used the free energy values corresponding with the reconstructions of the full ERP time window, this means from $100 \mathrm{~ms}$ before stimulus to $500 \mathrm{~ms}$ after stimulus. This resulted in 3 different free energy values for each of the 12 subjects and each of the stimulus conditions. Bayesian model selection was used to identify the best model using 4 measures: the log group Bayes factor, the expected posterior model frequencies, the exceedance probability and the Bayesian omnibus risk (BOR). These measures are described in mathematical detail in Stephan et al. (2009) and Rigoux et al. (2013). To compare the considered models, the free energy of the reconstructions were grouped over stimulus conditions, meaning that we compared 48 free energy values for each of the models.

We performed a fixed effects analysis at the group level. This is accomplished by calculating the log group Bayes factor which is the sum across subjects of individual log Bayes factors (Stephan et al., 2009), see (Strobbe et al., 2014) for more details. According to a decision rule described in Penny et al. (2004) one model can be chosen in favor of the other when there is a difference larger than 3 . 
We also performed a random effects analysis and reported the expected posterior model frequency, exceedance probability and the BOR. The expected posterior model frequency reflects the proportion of participants that favor a certain model. The exceedance probability expresses belief that a model has the highest posterior probability, relative to the other model. The BOR directly quantifies the probability that the expected posterior model frequencies are all equal to each other. If the BOR is smaller than 0.25 , then we can be confident in choosing the best model based on the results of the exceedance probability.

\subsubsection{Comparison of the reconstructed activity}

To check the validity of the reconstructions based on the different models, we compared the evoked energy of the ERP reconstructed activity corresponding with the P1-peak. We used time windows of $16 \mathrm{~ms}$ centered around the peak. In Fig. 4 an example is given of the ERP waveforms in each of the stimulus conditions for one of the subjects on a left and right lateral occipital channel. The time windows for which we calculated the evoked energy are depicted by the different colors.

For the volumetric approaches, the subject specific volumetric source activity was transformed to MNI space, based on a spatial normalization transformation used to normalize the MR images to the MNI template in SPM8 (Friston et al., 1996). Because a canonical cortical mesh in MNI space was used warped to the subject's anatomy to construct the cortical patches, each source of the mesh in subject space was directly corresponding with a location in MNI space (Mattout et al., 2007). As such, we generated 3D images corresponding with the P1 activity in MNI space. We averaged the result- 
ing images over subjects for each condition to compare the mean evoked P1 energy for each of the models.

From previous studies with similar ERP waveforms, we know that P1activity is mainly generated contralateral to the stimulus, located around the calcarine sulcus, in the fusiform gyrus and lingual gyrus (see (Novitskiy et al., 2011) and (Mijović et al., 2012)). These findings are used to compare the reconstructed activity based on the different models and for the different stimulus conditions (see Section 3.2).
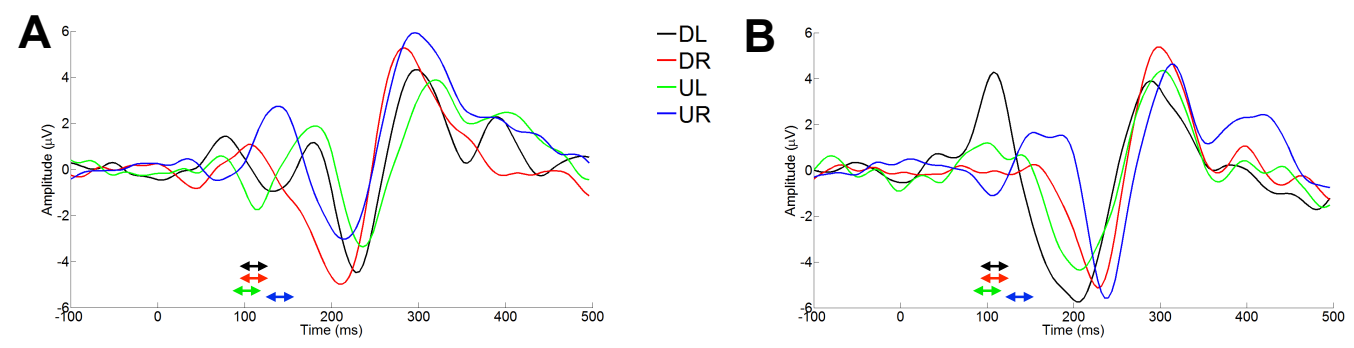

Figure 4: ERP data averaged over trials of one of the subjects for a (A) left lateral occipital and a (B) right lateral occipital channel. $\mathrm{DL}=$ down left stimuli, $\mathrm{DR}=$ down right stimuli, $\mathrm{UL}=$ upper left stimuli, $\mathrm{UR}=$ upper right stimuli. The arrows depict the time windows in which we calculated the mean evoked energy across subjects, 100 - 116 ms for DL stimuli, 100 - $116 \mathrm{~ms}$ for DR stimuli, 92 - $108 \mathrm{~ms}$ for UL stimuli and 132 - $158 \mathrm{~ms} \mathrm{~ms}$ for UR stimuli.

\section{Results}

\subsection{Bayesian model comparison}

In Fig. 5, the log Bayes factors are shown, computed as differences in free energy (F) corresponding with the reconstructions based on the considered 
models for each stimulus condition and every subject. In the first row, we compared the 3-layered models assuming volumetric regions, $\mathrm{Vol}_{3 l a y}$, with the 3-layered models assuming cortical surface patches, Surf ${ }_{3 l a y}$. In the second row, the 4-layered models assuming volumetric regions, $V_{\text {llay }}$ were compared with the $\mathrm{Surf}_{3 \text { lay }}$ models. The $\mathrm{Vol}_{4 l a y}$ models were compared versus the $\mathrm{Vol}_{3 l a y}$ models in the last row. We can notice a trend in favor of the $V_{1} l_{4 l a y}$ models compared to both 3-layered models and of the $\mathrm{Vol}_{3 l a y}$ modes in favor of the Sur 3 lay models.

To verify these trends statistically over subjects and stimulus conditions, we performed Bayesian model selection for group studies. We performed two analysis resulting in summary statistics for model comparison. In the first analysis we compared the Surf 3 lay and $\mathrm{Vol}_{3 l a y}$ models. The results are presented in the left panel of Fig. 6. In the first row, the log group Bayes factor is shown calculated versus the 3-layered volumetric models. It is clear it is considerably lower than -3 , which corresponds with very strong evidence in favor of the model assuming volumetric regions. The expected posterior model frequency and exceedance probability confirm this with an exceedance probability of the volumetric models equal to 0.99 . The BOR for this comparison is equal to 0.018 , which is below 0.25 and suggests that we can be very confident about the results of the exceedance probability.

In the second analysis we also included the $\mathrm{Vol}_{4 l a y}$ models. The results are shown in Fig. 6 on the right panel. The first row shows the log group Bayes factor calculated versus the 4-layered volumetric models. Both 3-layered models have log group Bayes factors much lower than -3 , which corresponds with very strong evidence in favor of the 4-layered volumetric model. The 


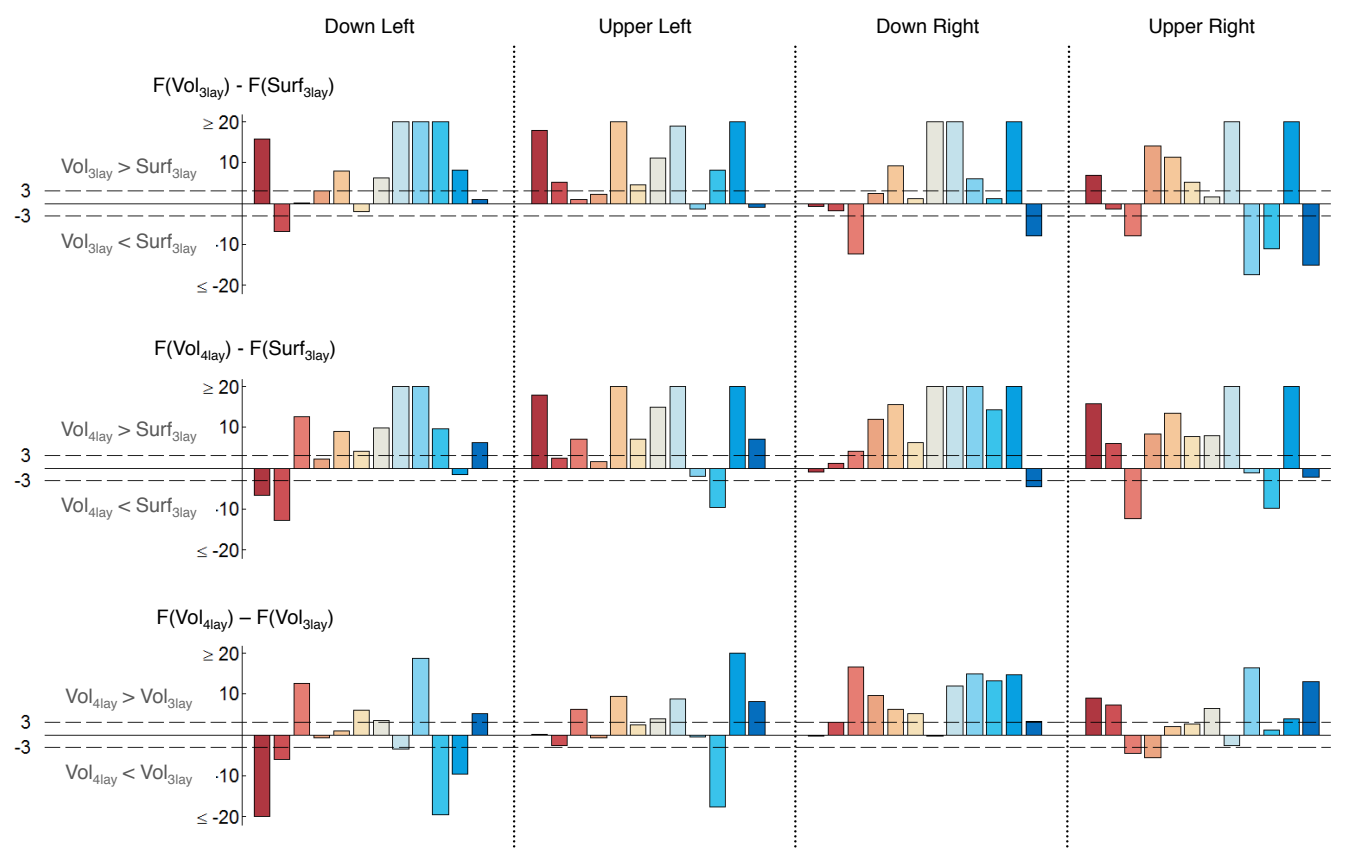

Figure 5: $\log$ Bayes factors computed as differences in free energy (F) to compare the considered models across subjects and stimlus conditions: Surf $_{3 l a y}$, for the 3-layered surface based models, $\mathrm{Vol}_{3 l a y}$, for the 3-layered volumetric models and $\mathrm{Vol}_{4 l a y}$ for the 4-layered volumetric models. The different subjects are represented by the different colors and each column represents a different stimulus condition. We used the $<$ and $>$ signs when there were differences bigger than 3 to denote evidence in favor of a one of the models. When comparing $\mathrm{Vol}_{3 l a y}$ versus Surf 3 lay for example, a difference bigger than 3 corresponds with very strong evidence in favor of the $\mathrm{Vol}_{3 \text { lay }}$ model, indicated as $\mathrm{Vol}_{3 \text { lay }}>\mathrm{Surf}_{3 l a y}$ on the left side of the bar graph. We restricted the y-axis to differences bigger than 20 to increase the interpretation of smaller differences. 
exceedance probability of the 4-layered model is equal to 0.99. With a BOR of 0.001 we found very clear evidence in favor of the 4-layered volumetric model.

\subsection{Comparison of the reconstructed activity}

In the first row of Fig. 7, maximum intensity projections are shown of the 99th percentile mean evoked energy across subjects corresponding with the P1 peak for the down left stimulus condition shown in different columns for the $\mathrm{Surf}_{3 l a y}, \mathrm{Vol}_{3 l a y}$ and $\mathrm{Vol}_{4 l a y}$ model. To enhance interpretation of the location of the reconstructed activity, the second row depicts the 99th percentile mean evoked energy rendered on the canonical cortical mesh in 2 views.

For the reconstructions based on the Surf 3 lay models, the activity is spread above the right calcarine sulcus, right lingual gyrus and right cuneus. Assuming both volumetric models, we found clear focal activity above the right calcarine sulcus and in the lingual gyri.

For both volumetric models, the P1 reconstructed activity agrees very well with the P1-activation found in a previous EEG/fMRI study using the same ERP data based on a jointICA decomposition (see Mijović et al. (2012) for more details). The late P1-activity that was found is included in the third row depicted in 3 orthogonal slices. The correspondence for the reconstructions

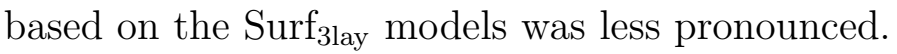

The maximum intensity projections for the other stimulus conditions are shown in Fig. 8. For the down right stimuli, the reconstructed activity based on $\mathrm{Vol}_{\text {llay }}$ was located above the left calcarine sulcus, left lingual gyrus and left fusiform gyrus. Assuming $\mathrm{Vol}_{\text {3lay }}$ the reconstructed activity was less lateralized. We found a strong activation in the right parahippocampal region. 

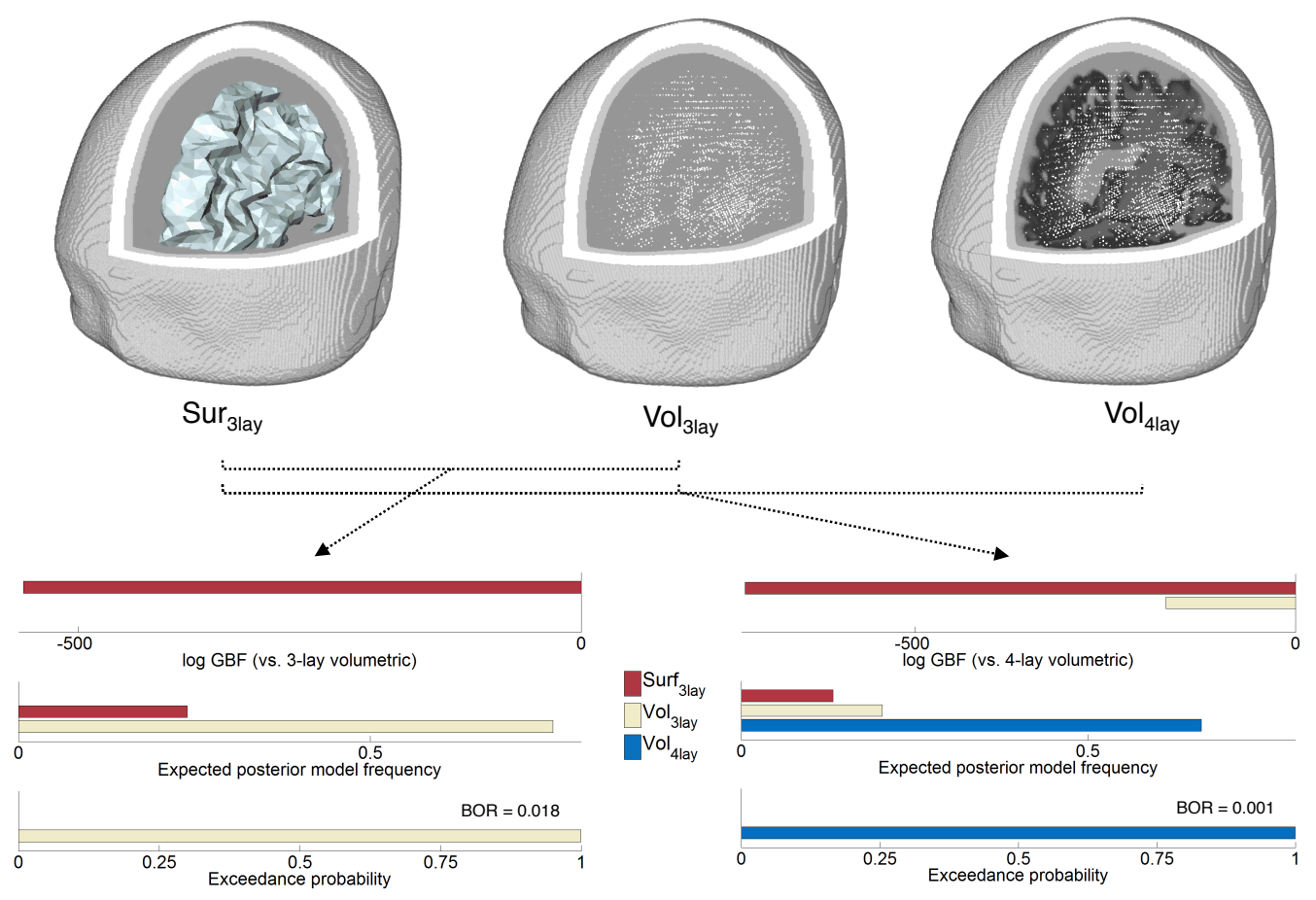

Figure 6: Bayesian model comparison results of the different models we assumed for reconstructing the ERP data: $\operatorname{Surf}_{3 l a y}, \mathrm{Vol}_{3 l a y}$ and $\mathrm{Vol}_{4 l a y}$. On the left, the comparison results are shown between the 3-layered models. On the right, the comparison results are shown for both the 3-layered models and the 4-layered volumetric models. For both comparisons, we show the log group Bayes factor (log GBF), the expected posterior model frequency and the exceedance probability. The different models we used for reconstruction are depicted by the different colors shown in the legend. The log GBF is calculated versus $\mathrm{Vol}_{3 l a y}$ for the comparison on the left and versus $\mathrm{Vol}_{4 l a y}$ for comparison on the right. We also show the Bayesian omnibus risk (BOR). If the BOR is smaller than 0.25, then we can be confident in choosing the best model based on the results of the exceedance probability. 

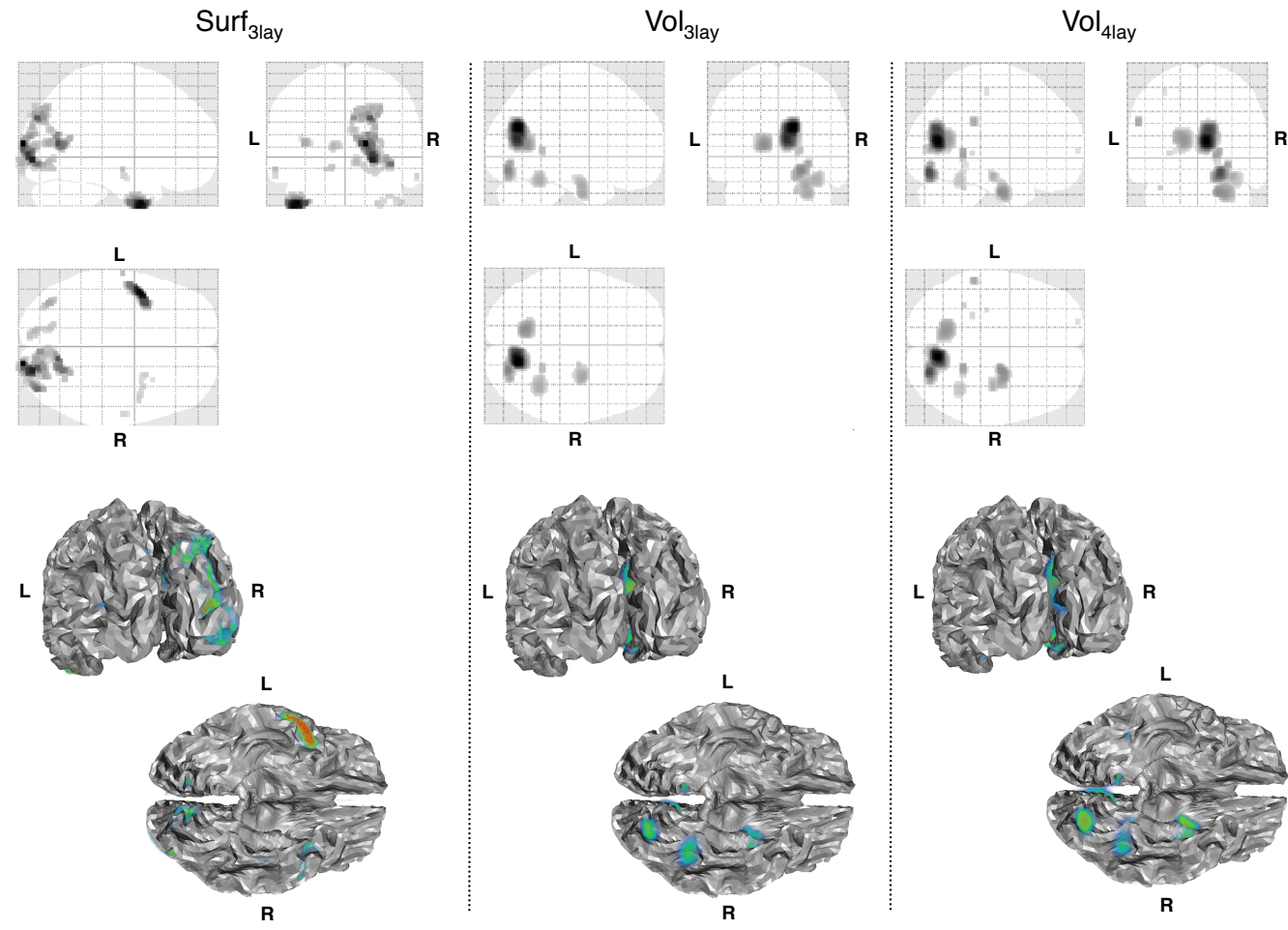

Mijovic et al. (Neurolmage 2012)

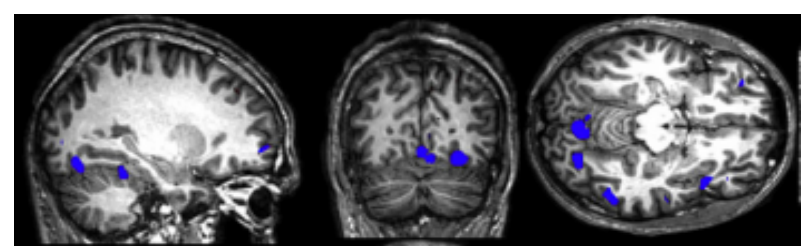

Figure 7: Maximum intensity projections of the 99th percentile of the mean evoked energy across subjects for the MSP inversions corresponding with the P1-peak (see Section 2.4.5) assuming Surf 3 lay, $\mathrm{Vol}_{3 l a y}$ or $\mathrm{Vol}_{4 l a y}$ depicted in the different columns. In the second row, 2 views are shown of the 99th percentile mean evoked energy rendered on the canonical cortical mesh. In the third row, the late P1-activity is shown from a jointICA EEG/fMRI decomposition reprinted from NeuroImage, Mijović et al. (2012), Copyright 2012, with permission from Elsevier. 
The reconstructed activity based on Surf 3 lay was mainly located in the left lingual gyrus, left inferior occipital gyrus, left midcingulate cortex, right precuneus and right fusiform region.

For the upper left stimuli, the reconstructed activity based on $\mathrm{Vol}_{4 l a y}$ was mainly located in the right lingual and right fusiform gyrus. For $\mathrm{Vol}_{3 l a y}$ the reconstructed activity was less lateralized with strong activation around the left calcarine sulcus. The reconstructed activity based on Surf 3 lay was mainly located in the right inferior occipital and temporal gyrus and the left inferior temporal gyrus.

For the upper right stimuli, the reconstructed activity based on $\mathrm{Vol}_{4 l a y}$ and $\mathrm{Vol}_{3 l a y}$ was mainly located around the left calcarine sulcus. The reconstructed activity based on Surf 3 lay was more widespread with the strongest activation located in the left cuneus.

\section{Discussion}

We have extended the current application of the multiple sparse priors algorithm (Friston et al., 2008) from sparse surface based priors to volumetric sparse regions. This extension provides the ability to use the MSP algorithm to reconstruct brain structures besides the cortical surface and opens up the ability to introduce more advanced volumetric head models based on volumetric forward modeling approaches using finite differences (Vanrumste et al., 2001), finite elements (Wolters et al., 2002) or finite volume modelling (Pruist et al., 1993). Both the Bayesian model selection analysis and the comparison of the reconstructed P1 activity demonstrated the value of the extension compared to the currently used approach. 


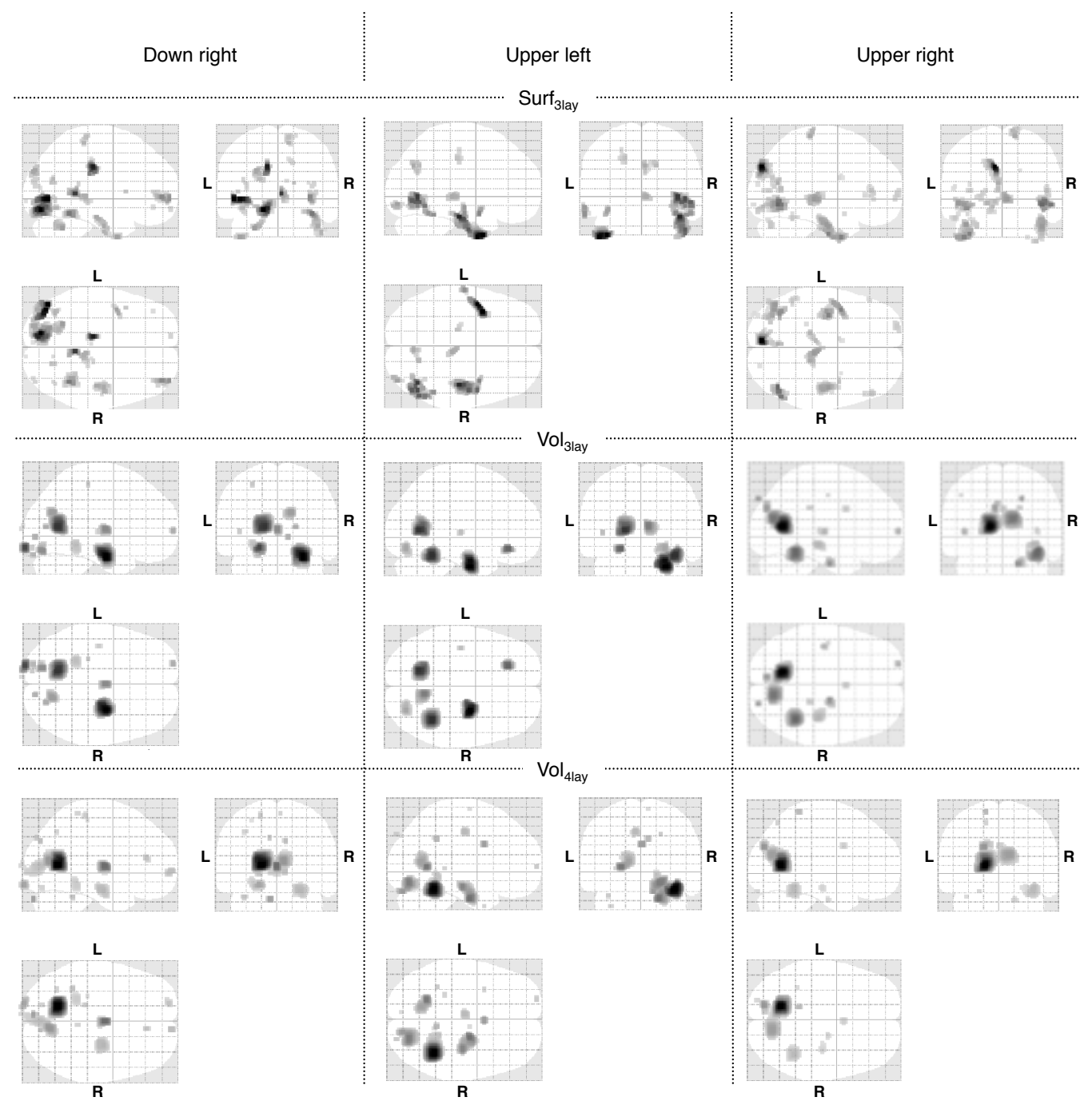

Figure 8: Maximum intensity projections of the 99th percentile of the mean evoked energy across subjects for the MSP inversions of the P1-peak (see Section 2.4.5) assuming Surf 3 lay, $\mathrm{Vol}_{3 l a y}$ or $\mathrm{Vol}_{4 l a y}$. The columns correspond with the different stimulus conditions 
Because we could not rely on a ground truth, we could not explain the differences in reconstructed activity quantitatively. However, we did find clear differences between the reconstructed activity assuming each of the models. By using maximum intensity projections of the P1 reconstructed activity for down left stimuli and showing this activity on the canonical cortical surface mesh we showed a high correspondence of the reconstructions based on the volumetric models with the findings of previous studies for down left stimuli (Novitskiy et al., 2011; Mijović et al., 2012). This was less pronounced assuming the surface based models. Also the reconstructions for the other stimulus conditions showed clear differences assuming each of the considered models. In general, the reconstructed activity assuming $\mathrm{Vol}_{4 l a y}$ was consequently focused in occipital regions contralateral to the stimulus which is congruent with previous studies in literature. This was less expressed for the reconstructions based on the 3-layered models. Notice that we would expect the activity for the upper stimuli to be more focused below the calcarine sulcus contralateral to the stimulus. Because the P1-components for the upper stimuli were not as highly expressed across subjects compared to the down stimuli we found deviations from the expected retinotopic locations in each of the models.

We did not compare the considered models with the currently used approach based on cortical patches and assuming a 4-layered head model including CSF because of the fact that the dipoles are located on the boundary between the CSF and brain compartment. Because we used a finite difference approach with a dipole model extending over 3 nodes of each voxel (the outer nodes have opposite currents (monopoles) in x, y or z direction (Hallez et al., 
2005)), the dipoles placed onto the CSF-brain boundary will have one side of the dipole source feeding directly to the CSF which is physically wrong and may have a large effect. We therefore had to ensure that at least 2 voxels of gray matter were between the central node of the dipole model and the boundaries with other tissues in the $\mathrm{x}, \mathrm{y}$ and $\mathrm{z}$ direction, in order to have a proper source model. To construct such a model based on the cortical surface mesh is very difficult because the mesh would need to be resampled, inflated or deflated to ensure no dipoles were modeled inside the CSF. With the extension proposed in this work, we could avoid this.

Note that we found evidence in favor of the $\mathrm{Vol}_{3 l a y}$ models compared to

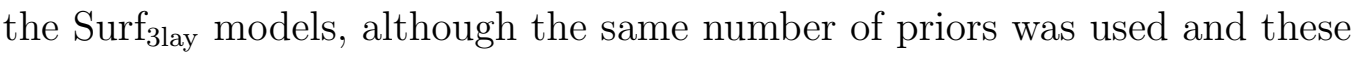
were at approximately the same cortical locations. The modeling of the volumetric regions was however closer to the actual anatomy of the subjects. They were constructed based on the actual segmented gray matter using the subject's anatomical MR image. For the construction of the cortical surface patches, a canonical cortical surface mesh was used which was warped to the subject's anatomy. This warping process is not faultlessly and the resulting cortical surface does not necessarily fully overlap with the actual cortical surface itself. Therefore also the orientations of the considered dipoles were different. For the dipoles located inside the gray matter layer, the orientations were determined based on the curvature of the segmented white matter and interpolating for gray matter. For the dipoles located on the cortical surface mesh, the orientations were set orthogonal to the surface. We could have used an approach in which we determined the individual cortical surfaces for each of the subjects based on subject's anatomical MRI. This is however not 
how it is done in the present implementation and the construction of these cortical surfaces often requires manual intervention (Henson et al., 2009).

Clearly, there are many issues that we have not addressed in this technical note. There are for example a lot of different other possibilities to construct the volumetric regions by introducing fMRI prior regions, anatomical priors, different smoothing functions, etc. Also other parameters can be optimized. These include the spacing of the dipoles, the optimal number of regions, the spacing of the regions, the smoothness of the regions, the dipole orientations, etc. The question of which set of priors will work best in practice depends on the data, and the Bayesian framework is a useful tool to evaluate different sets of priors in future work.

Note that the free energy we used to compare models, only provides an approximation to the Bayesian log evidence (see Wipf \& Nagarajan (2009) for a detailed discussion). Based on almost 30 years of advances and successful results in the field we have enough confidence however that it provides a good measure to compare models (Friston et al., 2007; Stephan et al., 2009; Penny, 2011). Moreover, we have no absolute information of how good the models are, but only which model is more probable depending on the data. In this context, future works including more complex models are highly recommended to be tested using the proposed framework.

We think that the use of realistic volumetric regions using advanced volumetric forward models can further improve PEB-EEG source reconstruction. In future studies, volumetric brain activity could be reconstructed based on volumetric regions including more anatomical or functional information. For example using cortical parcellation information (Knösche et al., 2013). Also 
future studies including more realistic head models should be introduced in the framework. For example, with accurate modeling of the skull including compact bone and spongy bone (Montes-Restrepo et al., 2013) or tissue anisotropy (Hallez et al., 2007). An important issue here is to assign the conductivity values to the different modeled tissues in the head model. The skull conductivity for example is strongly debated. Assuming a lower conductivity, for example $0.0041 \mathrm{~S} / \mathrm{m}$ assumed in the Fieldtrip software package (Oostenveld \& Oostendorp, 2002), would cause differences in source depths (Dannhauer et al., 2011; Montes-Restrepo et al., 2013). Again the Bayesian framework is a useful tool to evaluate different modeling options based on realistic data.

A drawback of using volumetric forward modeling techniques could be the computation time. In this study, the FDRM computation was done in parallel on a HPC cluster. Because of the reciprocity principle the computation was parallelized for each electrode pair (Vanrumste et al., 2001). It took about 10 minutes to calculate a subject specific model. Regarding the increasing computer power and memory this should not be an issue for future studies.

\section{Software note}

The described methods in this technical note are freely available. Please contact the authors for the scripts. We are currently working on a toolbox to integrate volumetric forward models into the SPM framework. 


\section{Acknowledgements}

Research supported by Research Council KUL: GOA MaNet, PFV/10/002 (OPTEC), by Flemish Government FWO projects: G.0427.10N (Integrated EEG-fMRI), by IWT- TBM080658-MRI (EEG-fMRI), iMinds, by Belgian Federal Science Policy Office: IUAP P7/(DYSCO, 'Dynamical systems, control and optimization', 2012-2017). This work was carried out using the Stevin Supercomputer Infrastructure at Ghent University, funded by Ghent University, the Hercules Foundation and the Flemish Government department EWI

\section{References}

Ashburner, J., \& Friston, K. (2003). Image segmentation. In R. Frackowiak, K. Friston, C. Frith, R. Dolan, K. Friston, C. Price, S. Zeki, J. Ashburner, \& W. Penny (Eds.), Human Brain Function. Academic Press. (2nd ed.).

Baillet, S., \& Garnero, L. (1997). A Bayesian approach to introducing anatomo-functional priors in the EEG/MEG inverse problem. IEEE Trans Biomed Eng, 44, 374-385.

Baumann, S. B., Wozny, D. R., Kelly, S. K., \& Meno, F. M. (1997). The Electrical Conductivity of Human Cerebrospinal Fluid at Body Temperature. IEEE Trans Biomed Eng, 44, 220-223.

Dale, A., \& Sereno, M. (1992). Improved Localization of Cortical Activity By Combining EEG and MEG with MRI Cortical Surface Reconstruction. Journal of Cognitive NeuroScience, c. 
Dannhauer, M., Lanfer, B., Wolters, C. H., \& Knösche, T. R. (2011). Modeling of the human skull in EEG source analysis. Human brain mapping, 32, 1383-99.

Friston, K., Harrison, L., Daunizeau, J., Kiebel, S., Phillips, C., TrujilloBarreto, N., Henson, R., Flandin, G., \& Mattout, J. (2008). Multiple sparse priors for the M/EEG inverse problem. NeuroImage, 39, 1104-20.

Friston, K., Mattout, J., Trujillo-Barreto, N., Ashburner, J., \& Penny, W. (2007). Variational free energy and the Laplace approximation. NeuroImage, $34,220-34$.

Friston, K. J., Ashburner, J., Frith, C. D., Poline, J., Heather, J. D., \& Frackowiak, R. S. J. (1996). Spatial registration and normalization of images. Hum Brain Mapp, 189.

Grech, R., Cassar, T., Muscat, J., Camilleri, K. P., Fabri, S. G., Zervakis, M., Xanthopoulos, P., Sakkalis, V., \& Vanrumste, B. (2008). Review on solving the inverse problem in EEG source analysis. J Neuroeng Rehabil, 5,25 .

Hallez, H., Vanrumste, B., Grech, R., Muscat, J., De Clercq, W., Vergult, A., D'Asseler, Y., Camilleri, K. P., Fabri, S. G., Van Huffel, S., \& Lemahieu, I. (2007). Review on solving the forward problem in EEG source analysis. J Neuroeng Rehabil, 4, 46.

Hallez, H., Vanrumste, B., Van Hese, P., D'Asseler, Y., Lemahieu, I., \& Van de Walle, R. (2005). A finite difference method with reciprocity used to 
incorporate anisotropy in electroencephalogram dipole source localization. Phys Med Biol, 50, 3787-3806.

Hämäläinen, M. S., \& Ilmoniemi, R. J. (1994). Interpreting magnetic fields of the brain: minimum norm estimates. Medical and Biological Engineering and Computing, 32, 35-42.

Henson, R. N., Mattout, J., Phillips, C., \& Friston, K. J. (2009). Selecting forward models for MEG source-reconstruction using model-evidence. NeuroImage, 46, 168-176.

Henson, R. N., Wakeman, D. G., , V., Friston, K. J., \& Trujillo-barreto, N. J. (2011). A parametric empirical Bayesian framework for the EEG / MEG inverse problem : generative models for multi-subject and multi-modal integration. Front Hum Neurosci, 5, 1-16.

Knösche, T. R., Gräser, M., \& Anwander, A. (2013). Prior knowledge on cortex organization in the reconstruction of source current densities from EEG. NeuroImage, 67, 7-24.

Mattout, J., Henson, R. N., \& Friston, K. J. (2007). Canonical Source Reconstruction for MEG. Comput Intell Neurosci, $200 \%$.

Mijović, B., Vanderperren, K., Novitskiy, N., Vanrumste, B., Stiers, P., Van Den Bergh, B., Lagae, L., Sunaert, S., Wagemans, J., Van Huffel, S., \& De Vos, M. (2012). The "why" and "how" of JointICA: Results from a visual detection task. NeuroImage, 60, 1171-1185.

Montes-Restrepo, V., van Mierlo, P., Strobbe, G., Staeles, S., Vandenberghe, 
S., \& Hallez, H. (2013). Inuence of skull modeling approaches on EEG source localization. Brain Topography, published online.

Mosher, J. C., Leahy, R. M., \& Lewis, P. S. (1999). EEG and MEG: forward solutions for inverse methods. IEEE transactions on bio-medical engineering, 46, 245-59.

Novitskiy, N., Ramautar, J. R., Vanderperren, K., De Vos, M., Mennes, M., Mijovic, B., Vanrumste, B., Stiers, P., Van den Bergh, B., Lagae, L., Sunaert, S., Van Huffel, S., \& Wagemeans, J. (2011). The BOLD correlates of the visual P1 and N1 in single-trial analysis of simultaneous EEG-fMRI recordings during a spatial detection task. NeuroImage, 54, 824-835.

Oostendorp, T. F., Delbeke, J., \& Stegeman, D. F. (2000). The conductivity of the human skull: results of in vivo and in vitro measurements. IEEE transactions on bio-medical engineering, 47, 1487-92.

Oostenveld, R., \& Oostendorp, T. F. (2002). Validating the boundary element method for forward and inverse EEG computations in the presence of a hole in the skull. Human brain mapping, 17, 179-92.

Penny, W. D. (2011). Comparing Dynamic Causal Models using AIC , BIC and Free Energy. NeuroImage, 59.

Penny, W. D., Stephan, K. E., Mechelli, a., \& Friston, K. J. (2004). Comparing dynamic causal models. NeuroImage, 22, 1157-72.

Phillips, C., Mattout, J., \& Friston, K. J. (2007). Statistical Paramteric Mapping. Elsevier. 
Phillips, C., Mattout, J., Rugg, M. D., Maquet, P., \& Friston, K. J. (2005). An empirical Bayesian solution to the source reconstruction problem in EEG. NeuroImage, 24, 997-1011.

Phillips, C., Rugg, M. D., \& Friston, K. J. (2002). Anatomically Informed Basis Functions for EEG Source Localization: Combining Functional and Anatomical Constraints. NeuroImage, 16, 678-695.

Pruist, G. W., Gilding, G. H., \& Peters, M. J. (1993). A comparison of different numerical methods for solving the forward problem in eeg and meg. Physiol. Meas., 14, A1-A9.

Rigoux, L., Stephan, K. E., Friston, K. J., \& Daunizeau, J. (2013). Bayesian model selection for group studies - Revisited. NeuroImage, 84C, 971-985.

Stenroos, M., \& Nenonen, J. (2012). On the accuracy of collocation and Galerkin BEM in the EEG / MEG forward problem. Int $J$ Bioelectromagn, $14,29-33$.

Stephan, K. E., Penny, W. D., Daunizeau, J., Moran, R. J., \& Friston, K. J. (2009). Bayesian model selection for group studies. NeuroImage, 46 , 1004-17.

Strobbe, G., van Mierlo, P., De Vos, M., Mijovic, B., Hallez, H., Van Huffel, S., Lopez, J., \& Vandenberghe, S. (2014). Bayesian model selection of template forward models for EEG source reconstruction. NeuroImage, .

Vanrumste, B., Van Hoey, G., Van de Walle, R., D’HAVE, M., Lemahieu, I., \& Boon, P. (2001). The validation of the finite difference method and 
reciprocity for solving the inverse problem in eeg dipole source analysis. Brain Topogr, 14, 83-92.

Wipf, D., \& Nagarajan, S. (2009). A unified Bayesian framework for MEG / EEG source imaging. NeuroImage, 44, 947-966.

Wolters, C. H., Kuhn, M., Anwander, A., \& Reitzinger, S. (2002). A parallel algebraic multigrid solver for finite element method based source localization in the human brain. Comp. Vis. Sci., 5, 165-177. 